\title{
ULTRASONIC ABSORPTION AND PERMEABILITY FOR LIPOSOMES NEAR PHASE TRANSITION
}

\author{
VALERIE M. MAYNARD, RICHARD L. MAGIN and FLOYD DUNN \\ Bioacoustic Research Laboratory, University of Illinois, 1406 West Green Street, Urbana, IL \\ 61801 (U.S.A.)
}

Received November $16 \mathrm{th}, 1984$ revision received February 11 th, 1985 accepted February 11 th, 1985

The specific ultrasonic absorption coefficient per wavelength as a function of temperature in the vicinity of the phase transition of liposomes, composed of a $4: 1$ mixture of dipalmitoylphosphatidylcholine (DPPC) and dipalmitoylphosphatidylglycerol (DPPG), of different sizes was determined using an acoustic interferometer. Small unilamellar vesicles (SUV) and multilamellar vesicles (MLV) yielded results similar to those in the literature, viz., an absorption maximum at the transition temperature. Seven intermediate sizes including several size distributions of large unilamellar vesicles (LUV) were studied, yielding information on size dependencies of the temperatures at which the peaks occur, the widths at half peak amplitude, and the peak amplitudes. All liposome sizes except the SUV exhibited approximately the same transition temperature as did the largest MLV. The widths of the peaks were inversely related to liposome size, with a strong dependence for the smallest vesicles and an approach to independence for the largest vesicles. The amplitudes of the peaks exhibited a general increase with size with two exceptions, viz., the SUV and the vesicles with average diameters of $90-100 \mathrm{~nm}$. It was also found that the membrane permeability increased near the transition temperature. The temperature dependencies of ultrasonic absorption and membrane permeability are compared.

Keywords. ultrasound; absorption; permeability; liposome; transition; size.

\section{Introduction}

The high frequency periodic variations of ultrasound can be used to investigate the mechanical [1] and thermodynamic [2] properties of biomembranes. Liposomes provide a convenient model for studying membrane structure and function [3]. The thermotropic phase transition of liposome membranes [4] has been studied using ultrasound. Near the transition temperature, a large increase in ultrasonic absorption occurs $[5,6]$. This temperature dependence is qualitatively the same as that exhibited by differential scanning calorimetry (DSC), a static thermodynamic technique whose results accurately reflect the extent of a thermal transition [4]. Ultrasonic studies not only illustrate pressure sensitive structural changes which accompany membrane phase transitions, but also have the potential to aid in the identification of dynamic events [7] of molecules or molecular groups. As a result of structural changes, the permeability of liposome membranes also increases dra- 
matically in the vicinity of the phase transition. Thus ultrasonic and permeability studies may contribute to a more complete understanding of membranes near the phase transition.

There are three categories of liposomes in terms of size and number of bilayers [8]. Multilamellar vesicles (MLV) exist in a rather wide range of sizes, but in general are the largest. Small unilamellar vesicles (SUV) are better defined morphologically, both in terms of having only one bilayer and having a narrower size distribution. However, their small radius of curvature may dictate physical and chemical behavior which is significantly different from that of vesicles with sizes on the order of those of biological cells [9]. Large unilamellar vesicles (LUV) are preferable to MLV and SUV as model membranes due to their single bilayer and large size, respectively [10]. LUV have been synthesized only more recently [11] and studied much less than MLV or SUV. Nevertheless, LUV have been manipulated successfully in several ways, including being forced into narrower size distributions [12]. This availability of various liposome diameters ranging from SUV through MLV makes possible a complete study of the effects of size on ultrasonic absorption.

Previous ultrasonic studies of the membrane transition have demonstrated the different behaviors exhibited by SUV and MLV [2]. Two studies of LUV have shown their behavior to be similar to that of MLV $[5,6]$. We report here the ultrasonic absorption behaviors near the transition temperature of nine morphologically different populations of liposomes of the same composition, covering a 25-fold diameter range. In addition, the temperature dependences of ultrasonic absorption and permeability are compared.

\section{Materials and Methods}

Dipalmitoylphosphatidylcholine (DPPC), and dipalmitoylphosphatidylglycerol (DPPG) were obtained from Avanti Polar Lipids (Birmingham, AL). Samples of DPPC and DPPG $(100 \mu \mathrm{g})$ gave single spots on thin layer chromatograms (silica gel $\mathrm{G}$ developed with $\mathrm{CHCl}_{3} / \mathrm{CH}_{3} \mathrm{OH} / \mathrm{H}_{2} \mathrm{O}, 64: 24: 4$, visualized with $\mathrm{I}_{2}$ vapor). Crystalline 1- $\beta$-D-arabinofuranosylcy tosine (Ara-C) and $N$-2-hydroxyethylpiperazine- $N^{\prime}-2$ ethanesulfonic acid (HEPES) were obtained from Sigma Chemical Company (St. Louis, MO). Tritiated cytosine arabinoside, $\left[{ }^{3} \mathrm{H}\right]-\mathrm{Ara}-\mathrm{C}(64 \mathrm{mCi} / \mathrm{mg}, 98 \%$ pure by thin-layer chromatography) and $\left[{ }^{14} \mathrm{C}\right] \mathrm{DPPC}(156 \mu \mathrm{Ci} / \mathrm{mg}, 98 \%$ pure by thin-layer chromatography) were obtained from Amersham Corporation (Arlington Heights, IL).

All liposomes were made from a mixture of DPPC/DPPG $(4: 1, w / w)$. The addition of DPPG, whose head group has a net negative charge at $\mathrm{pH} 7.4$, decreases liposome aggregation and presumably fusion. The phospholipids were mixed in chloroform (125 mg lipid/6.5 ml), which was then removed by a rotary evaporator. The procedure diverges at this point depending upon the type of liposome desired. A brief description of the liposome synthesis methods is given below; a more complete description of these procedures has been published [13-15]. 
To make MLV, the dried lipids are dissolved at $47^{\circ} \mathrm{C}$ (a temperature well above the transition temperature $\left(T_{m}\right)$ [16] of $42^{\circ} \mathrm{C}$ for bilayers comprising these phospholipids) in a solution of HEPES buffer (10 mM HEPES, $139 \mathrm{mM} \mathrm{NaCl}, 6 \mathrm{mM}$ $\mathrm{KCl}$ ), distilled water, Ara-C, and $0.1 \mathrm{~N} \mathrm{HCl}$. The buffer was diluted $1: 10$ with the water, the final solution concentration was brought to $300 \mathrm{mM}$ with Ara-C, a small water soluble molecule, and the $\mathrm{pH}$ was adjusted to 7.4 with $\mathrm{HCl}$. The liposomes were then formed in a vortex mixer, maintained at a temperature above $T_{m}$ for about $30 \mathrm{~min}$ and then cooled rapidly to room temperature. The suspension was exhaustively dialyzed at a low temperature against the HEPES buffer.

SUV were made by breaking MLV in a bath-type sonicator $(80 \mathrm{kHz}$, cavitating intensity, model G112SP1T, Laboratory Supplies Co., Hicksville, NY). The MLV suspension was sealed under nitrogen gas to prevent oxidation of the lipids and sonicated at a temperature between $45^{\circ} \mathrm{C}$ and $50^{\circ} \mathrm{C}$ until the suspension became transparent. After decreasing the temperature rapidly to below $T_{m}$, the suspension was placed in an ultracentrifuge to eliminate any remaining large vesicles.

LUV were made be the reverse phase evaporation process. After the initial mixing and drying, the lipids were redissolved in an organic solution of $8 \mathrm{ml}$ isopropyl ether and $4 \mathrm{ml}$ chloroform. At $47^{\circ} \mathrm{C}, 4 \mathrm{ml}$ of the buffer dilution described above were added. The mixture was sealed under nitrogen gas and sonicated for 5 min, forming an emulsion, which was then transferred to a rotary evaporator, where reverse evaporation, i.e., preferential removal of the organic phase, took place. The buffer dilution which evaporated was replaced. The suspension was then dialyzed at low temperature.

Different liposome size distributions were obtained by extrusion through one or more polycarbonate filters of successively smaller pore sizes $[12,14,17]$. The suspension was diluted to $8-10 \mathrm{mg} \mathrm{lipid} / \mathrm{ml}$, and the extrusion was conducted at $47^{\circ} \mathrm{C}$ by forcing the suspension through the filter by a syringe. MLV were successively extruded through filters with pore sizes of $0.8,0.6,0.4,0.2$ and $0.1 \mu \mathrm{m}$. LUV were successively extruded through filters with pore sizes of $0.4,0.2,0.08,0.05$ and 0.03 $\mu \mathrm{m}$. The extrusion process did not result in the loss of lipid, as monitored by $\left[{ }^{14} \mathrm{C}\right]$ DPPC activity. The percentage of captured solute decreased with polycarbonate membrane pore size for both MLV and LUV, as monitored by $\left[{ }^{3} \mathrm{H}\right]$-Ara-C activity, presumably due to the formation of smaller liposomes [17].

There is general agreement from many studies that SUV have a narrow size distribution, and that the average diameter is about $20 \mathrm{~nm}[18,19]$. The mean diameter of the large LUV was determined by low angle light scattering ( $\mathrm{N} 4$ submicron particle analyzer, Coulter Electronics, Hialeah, FL). The mean diameter of the large MLV was determined by transmission electron microscopy. The diameters of smaller LUV and MLV were estimated from the polycarbonate membrane pore sizes [8,17]. Olson et al. [17] have shown that when the pore diameter is reduced to about onethird of the mean diameter of the original liposome population, the mean diameter of the extruded population is approximately equal to the pore diameter.

For measurement of Ara-C release, the liposomes were diluted to approximately 
$10 \mathrm{mM}$ lipid with HEPES buffer. The suspension was transferred to a small glass bottle and placed in a circulating water bath at $35^{\circ} \mathrm{C}$. The temperature of the bath was increased in regular increments of $1^{\circ} \mathrm{C}$, allowing $5 \mathrm{~min}$ at each temperature. Aliquots $(100 \mu \mathrm{l})$ were removed at each temperature step and placed in $5 \times 20 \mathrm{~mm}$ cellulose propionate centrifuge tubes (Beckman Instruments, Palo Alto, CA) and centrifuged for $5 \mathrm{~min}$ at $178000 \times \mathrm{g}$ (Beckman Airfuge) to pellet the liposomes. A 50- $\mu$ l sample of the clear supernatant was tested for the presence of $\left[{ }^{3} \mathrm{H}\right]-\mathrm{Ara}-\mathrm{C}$, using a liquid scintillation counter (Beckman LS-7500). The percentage of Ara-C which was released was determined from these data. The $\left[{ }^{14} \mathrm{C}\right]$ DPPC in the supernatant was simultaneously measured to confirm that all liposomes were pelleted.

Samples for acoustic measurements were obtained by diluting a suspension with buffer to a lipid concentration of $2 \mathrm{mg} / \mathrm{ml}$ and placing about $4 \mathrm{ml}$ in a fixed-path, two-transducer, variable frequency, acoustic interferometer $[6,20]$. The absorption per wavelength is related inversely to the mechanical quality factor of the resonant cylinder as $\alpha \lambda=\pi \Delta f / f_{\mathrm{o}}=\pi / Q$, where $\alpha \lambda$ is the absorption coefficient per unit path length, $\lambda$ is the wavelength at resonance, $\Delta f$ is the difference in the frequencies greater than and less than the resonant frequency at which the average power has decreased to one-half that at resonance, $f_{\mathrm{o}}$ is the resonant frequency, and $Q$ is the quality factor describing the magnitude of resonance. The excess absorption per wavelength, i.e., that contributed by the liposomes, is obtained by subtracting the results of a reference measurement (the reference being the buffer in this case), which contains all of the contributions to the decrease of the quality factor except that due to the liposomes, i.e., $\alpha \lambda=\pi\left(\Delta f-\Delta f_{\text {ref }}\right) / f_{\mathrm{o}}$. The interferometer was suspended in a controlled temperature bath (Exacal 500 with Endocal 350 refrigeration unit, Neslab, Portsmouth, NH), which maintained the temperature stable to within $0.1^{\circ} \mathrm{C}$ while data were collected at a particular temperature, and to within $0.01^{\circ} \mathrm{C}$ while a particular bandwidth measurement was being made. The electrical input to the interferometer was supplied by a synthesized signal generator $(8660 \mathrm{~B}$. HewlettPackard). and the output was monitored by a spectrum analyzer (8552A, 8553B. Hewlett-Packard). The ultrasonic intensities used $\left(\sim 1 \mu \mathrm{W} / \mathrm{cm}^{2}\right)$ were orders of magnitude below those which can result in cavitation or in a significant temperature increase $121 \mid$. Thus. this was a passive ult rasonic study, i.e.. one designed to examine, not modify, a specimen. The excess specific absorption per wavelength ( $\pm 5 \%$ ) as a function of temperature $\left( \pm 0.05^{\circ} \mathrm{C}\right)$ was obtained at $3 \mathrm{MHz}$.

\section{Results and Discussion}

Both the static [22] and dynamic [23] behavior of the membrane transition have been shown to be a function of liposome size. The dynamic study [23] analyzed temperature-jump spectra of fluorescent intensity and, as such, dealt with a relatively slow process, viz., a process whose relaxation time varies from about $1-100$ ms, depending upon liposome size. Sano et al. [5] have shown that there is a single rapid process, one amenable to ultrasonic investigations. Previous ultrasonic studies reported more than one relaxation process, but liposome morphology was defined 
only to the extent of being MLV or SUV $[2,24]$. Sano et al. [5] studied two relatively precisely defined sizes, viz., SUV with a diameter of $21 \mathrm{~nm}$ and LUV with a diameter of $90 \pm 20 \mathrm{~nm}$. They concluded that different sized liposomes exhibit different relaxation times for the rapid process. In addition, they found that the behavior of these LUV in terms of the temperature at which the absorption peak occurs, its width, and its amplitude, was very similar to that of MLV. The present study shows that this behavior persists down to very small diameters. Table I tabulates these parameters for this study and for previous ultrasonic studies.

A general comparison of the temperature dependence of ultrasonic absorption characteristics for the three morphological categories of liposomes is illustrated by Fig. 1. The MLV and LUV data are for liposomes which have been extruded only once; i.e., they have the largest mean diameters in each category. The salient features include the similar temperature dependent absorption characteristics, in terms of both position and shape of the peaks, exhibited by MLV and LUV; the SUV peak occurs at a significantly lower temperature, is much broader, and has a smaller amplitude.

The temperature of maximum absorption indicates the melting temperature, $T_{m}$, of the membrane as revealed by the coincident peaks from ultrasound and DSC studies. For the SUV, $T_{m}$ obtained in the present study agrees with Sano et al. [5], but is almost $2^{\circ} \mathrm{C}$ less than the value reported by Harkness and White [2] (Table I).

TABLE I

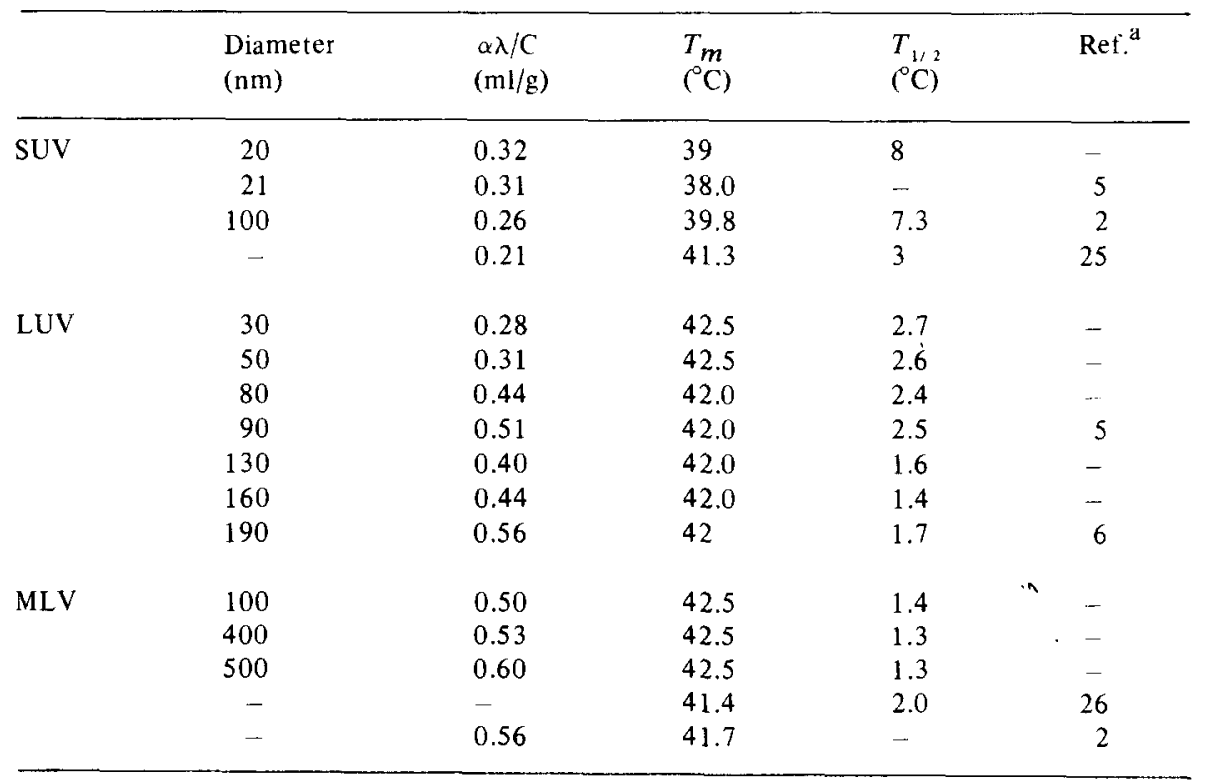

${ }^{a}$ When no reference is given, data are from this study. 


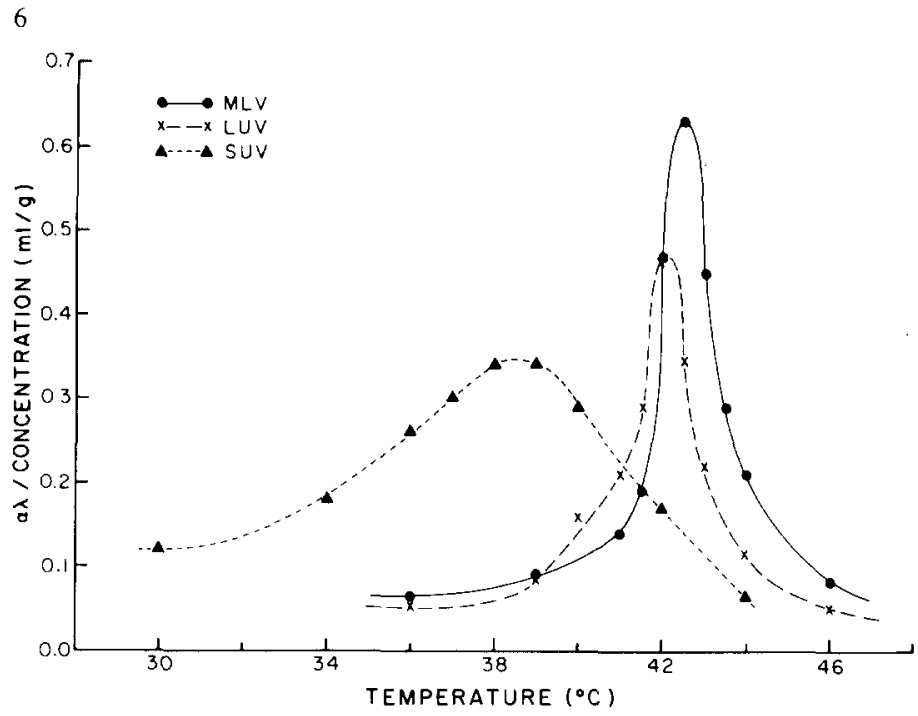

Fig. 1. Temperature dependence of the specific ultrasonic absorption coefficient per wavelength at $3 \mathrm{MHz}$ for suspensions of SUV, LUV and MLV. The LUV and MLV were extruded through $0.4 \mu \mathrm{m}$ and $0.8 \mu \mathrm{m}$ polycarborate membranes, respectively.

However, in the latter study, it is likely that the sample included many larger liposomes since a mean diameter of $100 \mathrm{~nm}$ was reported.

A graph of absorption versus temperature for the populations of the largest and smallest sized MLV is shown in Fig. 2. The larger MLV seem to be responsible for

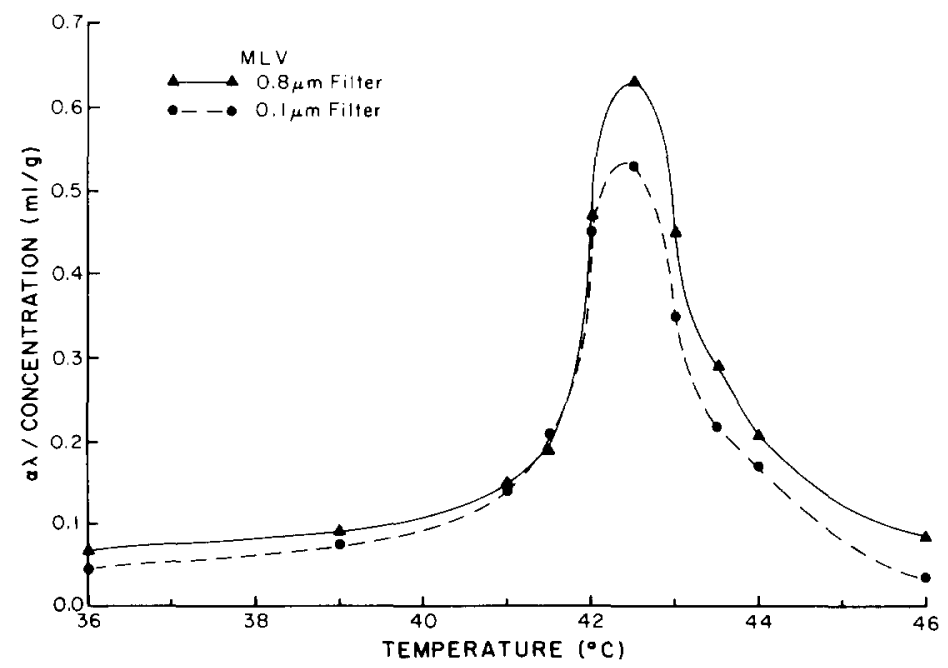

Fig. 2. Temperature dependence of the specific ultrasonic absorption coefficient per wavelength at $3 \mathrm{MHz}$ for suspensions of MLV. 
the absorption at slightly higher temperatures. However, $T_{m}$ is not affected significantly by the five-fold change in the mean diameter of these large liposomes. $T_{m}$ is about $1^{\circ} \mathrm{C}$ higher than that obtained by Harkness and White [2] and Mitaku and Okano [26]. DSC has yielded values of $41.5^{\circ} \mathrm{C}[27]$ and $43.5^{\circ} \mathrm{C}[4]$.

Figure 1 clearly shows that larger LUV have a $T_{m}$ slightly, but definitely, less than do MLV. This lower temperature confirms the trend with size suggested by the MLV data. The two populations of smallest LUV exhibit anomalous $T_{m}$-values in that they are the same as for the MLV. Thus if $T_{m}$ is a continuous function of size, there must be a very large functional change between approx. 20 and $30 \mathrm{~nm}$. If not, some qualitative difference between SUV and the smallest LUV results in a discontinuity in $T_{m}$. The $T_{m}$ of the midsized LUV agrees with the value obtained by Sano et al. [5] for a similar size population.

$T_{1 / 2}$, the width of the specific absorption versus temperature curve at one-half the height. for the SUV is similar to the value obtained by Harkness and White [2] (Table I), and agrees with the value $\left(8.0^{\circ} \mathrm{C}\right.$ ) obtained by Melchior and Steim [4] using DSC.

For MLV, $T_{1 / 2}$ is not affected significantly by the 5-fold change in diameter. $T_{1 / 2}$ obtained from the data of Mitaku and Okano [26] is somewhat larger than that obtained in the present study. DSC results vary even more. Those reported by Melchior and Steim [4] yield a value of $3^{\circ} \mathrm{C}$, while Suurkuusk et al. [28] report a value of $0.7^{\circ} \mathrm{C}$. DSC by Duzgunes et al. [10] yielded a value in agreement with that of the present study, $1.3^{\circ} \mathrm{C}$.

$T_{1 / 2}$ for the large LUV is the same as that of the MLV. SUV-type behavior qualitatively begins to appear as the LUV size decreases (Fig. 3). $T_{1 / 2}$ increases by a factor of two as the diameter decreases by a factor of four. The width of the curve for the LUV of the same size as that studied by Sano et al. [5] agrees with their value. However, the transition of the smallest LUV is still not nearly as broad as that of the SUV (Fig. 4).

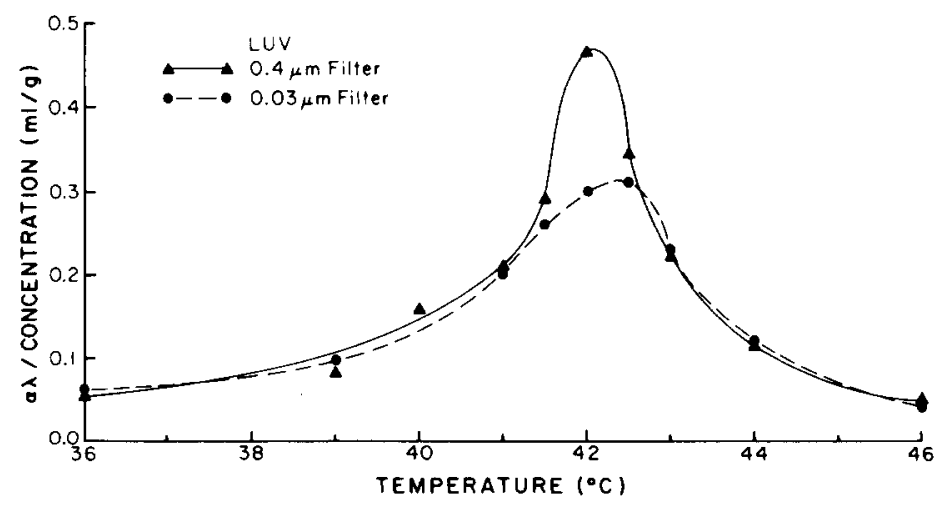

Fig. 3. Temperature dependence of the specific ultrasonic absorption coefficient per wavelength at $3 \mathrm{MHz}$ for suspensions of LUV. 


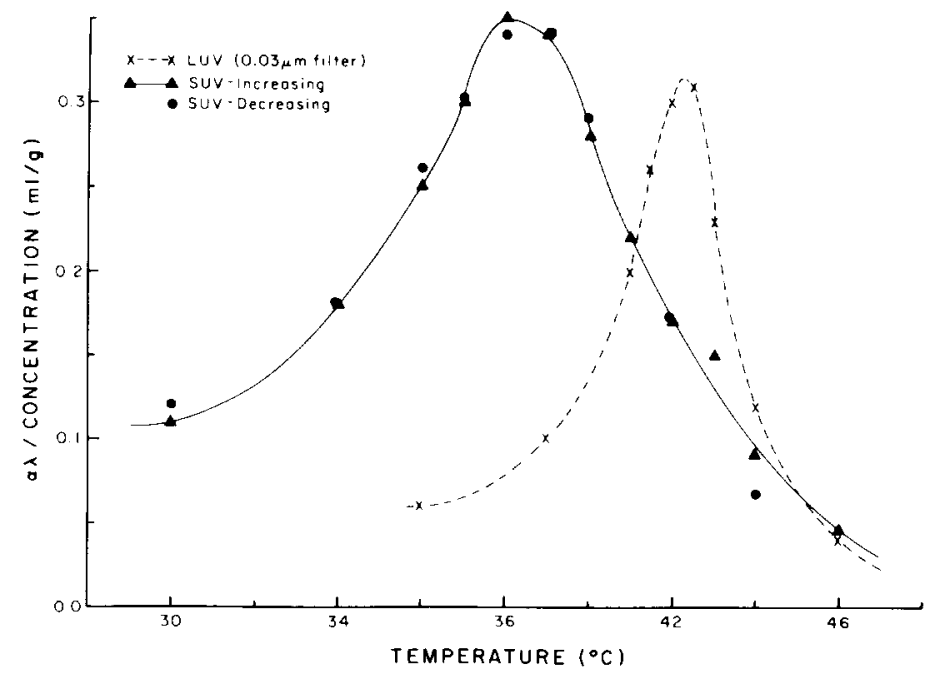

Fig. 4. Temperature dependence of the specific ultrasonic absorption coefficient per wavelength at $3 \mathrm{MHz}$ for suspensions of SUV and LUV.

It is unlikely that the significant differences in $T_{m}$ and $T_{1 / 2}$ between the SUV and the larger liposomes are due to the different methods of preparation. Other studies [29,30] have shown similar behavior for liposomes prepared by several methods. cach covering a small size range in the SUV--small LUV region. The somewhat more gradual change observed in these studies, compared with those of the present investigation, may be ascribed to the different methods employed for monitoring the phase transition.

The peak value of the specific absorption coefficient per wavelength is in essential agreement with that obtained by Sano et al. [5], but is larger than that obtained by Harkness and White [2] (Table I). By referring forward to Fig. 5, it can be seen that, considering the relatively large size of their SUV, a significantly larger absorption would be predicted.

The peak amplitude obtained for the largest MLV is slightly larger than the value shown by the results of Harkness and White [2]. It can be seen that, unlike $T_{m}$ and $T_{1 / 2}$, the peak amplitude is affected by the size change, although not greatly (Fig. 2). Except for this amplitude decrease, even the smallest MLV have not breached the morphological characteristics which result in the behavior of SUV.

The peak amplitudes for the LUV decrease abruptly between populations with mean diameters of 80 and $50 \mathrm{~nm}$. The large LUV yield amplitudes somewhat less than do the MLV of approximately the same size. The small LUV yield amplitudes approximately equal to that of the SUV (Fig. 4). From the data of Sano et al. [5]. the peak amplitude for LUV with a mean diameter of $90 \mathrm{~nm}$ was found to be somewhat larger than that which was obtained in the present study for similar sized liposomes. 
The characteristics of the SUV peak were independent of the direction of the temperature change (Fig. 4), indicating that, at least for those liposome characteristics to which ultrasound is sensitive, passing through the absorption peak does not result in a permanent change. Any hysteresis that may have been associated with the temperature change was completed in a time shorter than that in which the data were obtained. This finding is at variance with some results from the literature, which show different values upon reversing temperature. One such study [2] also found that the average diameter of the vesicles doubled upon cycling through the transition. The observed change was an increase in absorption only on the high temperature side of the peak. In another study [9]. repeated temperature cycling yielded a second peak at a higher temperature, specifically, that which is characteristic of MLV. The absorption amplitude grew linearly with the number of cycles at the expense of the SUV peak. Thus the nonrepeatability of the data indicates vesicle aggregation at the least, and possibly fusion. The absence of any such effect in this study suggests that the presence of the negatively charged DPPG has the desired effect of preventing such aggregation. This satisfactory behavior of SUV implies that the same is true for the larger vesicles, because even for those studies in which aggregation of SUV occurred, aggregation did not occur in large liposomes.

Changes in the parameters reported herein are not straightforward functions of the change in size of liposomes. The higher $T_{m}$ of the largest liposomes is maintained through the smallest LUV. The $T_{1 / 2}$ increases abruptly with decreasing diameter near $100 \mathrm{~nm}$ and again at the transition to SUV.

If the two critical diameters, in terms of abrupt parameter changes, of approx. 20 and $100 \mathrm{~nm}$ are excluded, a $\log \log$ plot of the peak amplitude data (Fig. 5) yields the following empirical relationship: $\alpha \lambda / C=0.1 d^{0.3} \mathrm{~cm}^{3} / \mathrm{g}$, where the left side is the specific absorption per wavelength, $C$ is concentration and $d$ is the lipo-

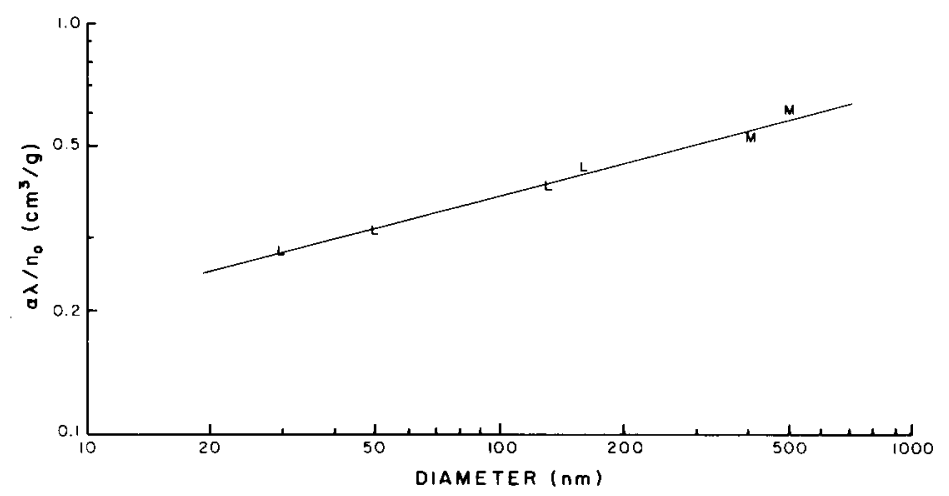

Fig. 5. Log-log plot of the dependence upon size of the specific ultrasonic absorption coefficient per wavelength at $3 \mathrm{MHz}$ for liposome suspensions. Data are labeled as: L for LUV and M for MLV. 
some diameter in nanometers. This may prove useful in predicting energy deposition in liposomes at greater ultrasonic intensities.

The permeability of the three classes of liposomes used in this study is illustrated by the release of $\left[{ }^{3} \mathrm{H}\right]$-Ara-C from liposomes at temperatures near $T_{m}$ (Fig. 6). The release of Ara-C from LUV increases sharply between $39^{\circ} \mathrm{C}$ and $40^{\circ} \mathrm{C}$, slightly below the $T_{m}$ of $42.0^{\circ} \mathrm{C}$. The MLV exhibit a broader release centered around $42^{\circ} \mathrm{C}$. The smaller measured drug release from MLV compared with LUV is probably due to the presence of multiple bilayers in the MLV, which must be crossed by the Ara-C. Unlike the MLV and LUV, which appear to be relatively impermeable below $T_{m}$, the SUV leak approx. 30\% of the Ara-C and show only a small increase in leakage as the temperature is increased through $T_{m}$. The basic drug release characteristics are consistent with previous studies [14,31].

The LUV and MLV used in the permeability studies have size distributions similar to those of the largest LUV and MLV used in the ultrasonic studies. Therefore, a direct comparison of the temperature dependencies of ultrasonic absorption and permeability can be made by referring to Figs. 1 and 6 . The similarity of LUV to MLV results and the dissimilarity to SUV results obtained in the ultrasonic studies is also the case for the permeability studies below $39^{\circ} \mathrm{C}$. At higher temperatures, the permeability of LUV is more similar to that of MLV in that both experience at least a several-fold increase, whereas SUV experience only a fractional increase. The decreased MLV drug release compared to that of LUV indicates the large effect which multiple bilayers have on permeability. The similar ultrasonic absorption exhibited by MLV and LUV indicates that this permeability difference is not due to some gross intramembrane structural difference between MLV and LUV. Rather,

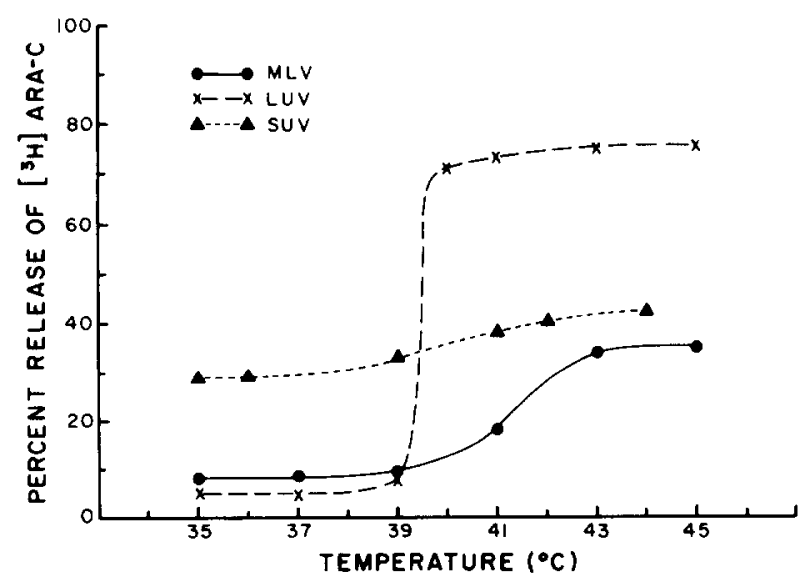

Fig. 6. Temperature dependence of the release of $\left[{ }^{3} \mathrm{H}\right]-\mathrm{Ara}-\mathrm{C}$ from MLV, LUV and SUV liposomes. 
it may be due to simply additional barriers or to a more subtle structural difference, subtle at least as far as ultrasound is concerned, caused by bilayer proximity.

The temperatures of maximum permeability increase for both LUV and MLV occur slightly below $T_{m}$. This temperature for LUV is $39.5^{\circ} \mathrm{C}, 2.5^{\circ} \mathrm{C}$ less than $T_{m}$, and for MLV is $41.5^{\circ} \mathrm{C}, 1.0^{\circ} \mathrm{C}$ less than $T_{m}$. When Fig. 1 is consulted for the extent of the transition which has occurred for LUV and MLV at $39.5^{\circ} \mathrm{C}$ and $41.5^{\circ} \mathrm{C}$, respectively, it is seen that only a small fraction of the transition need occur for these membranes to greatly increase their permeability to Ara-C. For LUV, if $T_{m}$ as indicated by the ultrasonic absorption peak is considered as marking $50 \%$ of the extent of the transition, then only about $7 \%$ of the transition need occur for there to be a large increase in Ara-C permeability. For MLV, this permeability increase occurs at slightly more than $10 \%$ into the transition. Again, however, this may be a multiple bilayer effect rather than an indication of intramembrane functional changes. The permeability increase of SUV occurs above the transition midpoint. Thus, the very different characteristics of SUV have been made manifest by both structural and functional studies.

These relationships between this probe of some physical characteristics of the membrane, viz., ultrasound, and an aspect of membrane function, viz., passive permeability, will be extended in future studies by the addition of different types of molecules in to the liposome membrane.

\section{Acknowledgements}

We are grateful to Michael Niesman for liposome synthesis. This work was supported by the following grants: 5 T32 CA09067 and CA 29010 of the National Cancer Institute, DHHS; GM12281 of the National Institutes of Health; 82-9 of the American Cancer Society, Illinois Division.

\section{References}

1 S. Mitaku, R. Kataoka, S. Aruga and T. Jippo, Il Nuovo Cimento, 3D (1984) 193.

2 J.E. Harkness and R.D. White, Biochim. Biophys. Acta, 552 (1979) 450.

3 M.K. Jain, in: Membrane Fluidity in Biology, Vol. 1, Academic Press, New York, 1983, Ch. 1.

4 D.L. Melchior and J.M. Steim, Annu. Rev. Biophys. Bioeng., 5 (1976) 205.

5 T. Sano, J. Tanaka, T. Yasunaga and T. Toyoshima, J. Phys. Chem., 86 (1982) 3013.

6 P.R. Strom-Jenson, R.L. Magin and F. Dunn, Biochim. Biophys. Acta, 769 (1984) 179.

7 F. Eggers and T. Funck, Naturwissenschaften, 63 (1976) 280.

8 F. Szoka and D. Papahadjopoulos, Annu. Rev. Biophys. Bioeng., 9 (1980) 467.

9 P.W.M. Van Dijck, B.de Kruijff, P.A.M.M. Aarts, A.J. Verkleij and J. de Gier, Biochim. Biophys. Acta, 506 (1978) 183.

10 N. Duzgunes, J. Wilschut, K. Hong, R. Fraley, C. Perry, D.S. Friend, T.L. James and D. Papahadjopoulos, Biochim. Biophys. Acta, 732 (1983) 289.

11 D. Deamer and A.D. Bangham, Biochim. Biophys. Acta, 443 (1976) 629

12 F. Szoka and D. Papahadjopoulos, Proc. Natl. Acad. Sci. U.S.A., 75 (1978) 4194. 
13 R.L. Magin and J.N. Weinstein, in: G. Gregoriadis (Ed.), Liposome Technology, Vol. III, CRC Press, Boca Raton, FL, 1984, Ch. 10.

14 R.L. Magin and M.R. Niesman, Chem. Phys. Lipids, 34 (1984) 245.

15 R.L. Magin and M.R. Niesman, Cancer Drug Delivery, 1 (1984) 109.

16 M.I. Kanehisa and T.Y. Tsong, J. Am. Chem. Soc., 100 (1978) 424.

17 F. Olson, C.A. Hunt, F.C. Szoka, W.J. Vail and D. Papahadjopoulos, Biochim. Biophys. Acta, 557 (1979) 9.

18 B. de Kruijff, P.R. Cullis and G.K. Radda, Biochim. Biophys. Acta, 406 (1975) 6.

19 J.T. Mason and C. Huang, Ann. N.Y. Acad. Sci. (1978) 29.

20 F. Eggers, Acustica, 19 (1968) 323.

21 W.J. Fry and F. Dunn, in: W.L. Nastuk (Ed.), Physical Techniques in Biological Research, Vol. 4, Academic Press, New York, 1962, Ch. 6.

22 H. Takemoto, S. Inoue, T. Yasunaga, M. Sukigara and Y. Toyoshima, J. Phys. Chem.. 85 (1981) 1032 .

23 S. Inoue, M. Nishimura, T. Yasunaga, H. Takemoto and Y. Toyoshima, J. Phys. Chem., 85 (1981) 1401 .

$24 \mathrm{~T}$. Funck and $\mathrm{F}$. liggers, Abstract in Program of Meeting of Fast Reactions in Solution Discussion Group, Chemical Society, London and Max-Planck Gesellschaft, 1980, p. 116.

25 R.C. Gamble and P.R. Schimmel, Proc. Natl. Acad. Sci., 75 (1978) 3011.

26 S. Mitaku and K. Okano, Biophys. Chem., 14 (1981) 147.

27 H.-J. Hinz and J.M. Sturtevant, J. Biol. Chem., 247 (1972) 3697.

28 J. Suurkuusk, B.R. Lentz, Y. Barenholz, R.L. Biltonen and T.E. Thompson, Biochemistry, 15 (1976) 1393.

29 P.W.M. Van Dijck, B. de Kruijff, P.A.M.M. Aarts, A.J. Verklij and J. de Gier, Biochim. Biophys. Acta., 506 (1978) 183.

30 S. Inoue, M. Nishimura and T. Yasunaga, J. Phys. Chem., 85 (1981) 1401.

31 J.N. Weinstein, R.L. Magin, M.B. Yatvin and D.S. Zaharko, Science, 204 (1979) 188. 